\title{
Evaluation of Mean Platelet Volume in Patients With Rheumatoid Arthritis and its Relation With Severity of Disease
}

\author{
Mandana Khodashahi ${ }^{1}$, Nayyereh Saadati ${ }^{1}$, Zahra Rezaieyazdi ${ }^{1}$, Maryam Sahebari ${ }^{1}$, Zeinab Saremi ${ }^{2 *}$ \\ ${ }^{1}$ Rheumatic Diseases Research Center, Mashhad University of Medical Sciences, Mashhad, Iran. ${ }^{2}$ Department of Inter- \\ nal Medicine, School of Medicine, Birjand University of Medical Science, Birjand, Iran.
}

\begin{abstract}
Rheumatoid arthritis (RA) is the most common chronic inflammatory disease of the joints. Inflammation is considered to be the main stimulant of platelets, and there is an association between the mean platelet volume (MPV) and platelet reactivity. Increased platelet counts may be observed during the clinical active stages of RA patients and reduced in the remission period. The present study investigated the role of platelet indices in RA and its relationship with disease activity.

This case-control study was performed on 105 RA patients and 35 healthy participants as the control group who referred to Ghaem Hospital of Mashhad in 2017. The participants were divided into 4 groups: 1) patients with newly diagnosed RA, 2) patients with active RA, 3) patients with RA in remission, and 4) healthy controls. After completing the interview and questionnaire, 10-cc blood samples were obtained from all subjects. The platelet number (PLT) and mean platelet volume (MPV) were measured.

The mean age of the participants was $49.06 \pm 11.08$ years. MPV was higher in patients with RA than in the healthy control group $(P$ value $<0.005)$. There was a significant difference in mean platelet volume $(\mathrm{MPV})$ between the remission and active groups $(P$ value $<0.05$ ).

MPV is higher in patients with RA than healthy control. Also is higher in patients with active RA than remission group.
\end{abstract}

Keywords: Disease Activity; Mean Platelet Volume; Platelet Number; Rheumatoid Arthritis

\section{Introduction}

Rheumatoid arthritis (RA) is one of the most common autoimmune diseases from which $0.5-1 \%$ of the world's population suffer [1] The incidence and prevalence rates of RA differ in various geographical areas as well as ethnic groups, and similar to other autoimmune diseases it is higher among women than men (female/male ratio: 3/2) [2]. This disease is accompanied by articular and extra-articular manifestations. Synovial inflammation, the production of anticitrullinated protein antibody, and the destruction of cartilage and bone are the most important basic features of RA [3].

Synovial membrane inflammation affects the small and large joints and leads to the incidence of synovial proliferative disorders and, consequently, cartilage destruction and ankylosis. Because cartilage destruction causes structural damage to the joints and ultimately, individual disability, early diagnosis and appropriate treatment for RA is very important [3].

To the best of our knowledge, the main role of platelets is the maintenance of hemostasis; however, they also play a critical role in inflammation and immune responses, which may be attributed to their role in the hemostasis process [4]. Based on evidence, host immunity is affected by antiplatelet medications, such that it is associated with a decrease in mortality due to sepsis and with reduced bleeding [5-7]. The evaluation of the role of platelets in inflammation and immune responses is very important because of the clinical benefits of antiplatelet agents in systemic inflammation related to infection and justifies detailed examination [8].

Several studies have identified the association between RA and markers of systemic inflammation, such as C-reactive protein (CRP), erythrocyte sedimentation rate (ESR), mean platelet volume (MPV), and tumor necrosis factor-alpha (TNF- $\alpha$ ) [9-12] and demonstrated that these factors are increased in RA patients. Inflammation is con-

Personal non-commercial use only.Rheumatology Research Journal. Copyright (C) 2019. All rights reserved

*Corresponding Author: Zeinab saremi. Internal medicine department,valiasr hospital. Birjand University of Medical Sciences. Birjand, Iran, Email: zsaremi@bums.ac.ir, Tel: (+98)9155340380

Received: 05 September 2019; Accepted: 30 October 2019 
sidered to be a main stimulant of platelets, and there is an association between the mean platelet volume (MPV) and platelet reactivity. Increased platelet counts may be observed during the clinical active stages of RA patients, and they are reduced in the remission period by different treatment modalities [13-15]. Few studies have investigated MPV values in RA patients and their relationship with disease activity $[15,16]$. Therefore, the present study aimed to investigate the role of platelet indices in RA and their relationships with disease activity.

\section{Materials and Methods}

This case-control study was carried out on 105 patients with RA and 35 persons as healthy control who referred to Ghaem Hospital in Mashhad in 2017. All patients met the ACR/EULAR 2010 criteria for the diagnosis of RA. The exclusion criteria were pregnancy; renal, liver, or heart failure; cancer and chemotherapy; acute or chronic infection, such as tuberculosis and brucellosis; and other simultaneous rheumatoid diseases (e.g., lupus, inflammatory myopathies, scleroderma, and Sjogren's syndrome). Participants were selected by systematic random sampling.

Patients in this study were divided into four groups: 1) subjects with newly diagnosed RA and no treatment, 2) cases with active RA (Disease Activity Score 28 [DAS28ESR]>3/2), 3) patients in remission (DAS28-ESR < 2.6) who received fixed treatments during the preceding two months, and 4) the controls who were matched with the other three groups in terms of age and gender (35 participants in each group).

All patients in the case and control groups were visited by a rheumatologist, and physical examinations were performed. After an interview and questionnaire completion, 10-cc blood samples were obtained from all subjects. Erythrocyte sedimentation rate (ESR), C-reactive protein (CRP), white blood cell count (WBC), platelet number (PLT), MPV, and hemoglobin (Hb) levels were measured. The diagnosis of RA activity was evaluated based on DAS28 ESR criteria.

\section{Statistical Analysis}

The descriptive data was entered into a code sheet and then analyzed in SPSS software (version 16). The Kolmogorov-Smirnov test was used to evaluate the normality of data. The qualitative and quantitative variables were analyzed using the chi-square test and Kruskal-Wallis test, respectively.

\section{Ethical Considerations}

The study protocol was approved by the Ethics Committee of Mashhad University of Medical Sciences, Mashhad, Iran (IR.MUMS.fm.REC.1396.149). In line with the principals of research ethics, informed consent was obtained from all patients, and all of them were assured that their information will remain confidential. The participants were ensured that they could withdraw from the study at any time.

\section{Results}

The mean age of the participants was $49.06 \pm 11.08$ years. About $89.29 \%(n=125)$ and $10.71 \%(n=15)$ of the patients were female and male, respectively. Table 1 tabulates the frequency of demographic information in the four groups. No significant difference among the four groups was observed in sex distribution $(P$ value $=0.87)$ or age distribution $(P$ value $=0.08)($ Table 1$)$.

The duration of treatment was less than one year in $8.7 \%$ and $7 \%$ of active RA patients and RA patients in remission, respectively. The duration of treatment in $31.42 \%$ of active RA patients and $34.28 \%$ of patients in remission was between 1-5 years. Moreover, the duration of treatment was more than five years in $60 \%$ and $45.71 \%$ of active RA patients and RA patients in remission, respectively. The comparison of treatment duration between active RA patients and RA patients in remission showed that there was no significant difference between them.

About $16.19 \%(n=17)$ and $11.4 \%(n=4)$ of RA patients and controls had a family history of RA, respectively. The comparison of the family history of RA patients and healthy controls showed that there was no significant difference between them $(P$ value $=0.49)$.

The comparison of MPV between the RA group and the healthy control group showed that there was a significant difference between them $(P$ value $=0.01)$; MPV was higher in the RA group. The comparison of platelet numbers between the RA group and the healthy control group

Table 1. Frequency of demographic information in four groups

\begin{tabular}{cccccc}
\hline Variables & $\begin{array}{c}\text { RA } \\
\text { ( remission) }\end{array}$ & $\begin{array}{c}\text { RA } \\
\text { (new) }\end{array}$ & $\begin{array}{c}\text { RA } \\
\text { (active) }\end{array}$ & Control & $P$ value \\
\hline age (years) & $48.11 \pm 11.76$ & $49.49 \pm 13.31$ & $52.88 \pm 10.1$ & $51.31 \pm 10.54$ & 0.08 \\
male (\%) & $4(11.43 \%)$ & $4(11.43 \%)$ & $3(8.57 \%)$ & $4(11.43 \%)$ & 0.87 \\
female (\%) & $31(88.57 \%)$ & $31(88.57 \%)$ & $32(91.43 \%)$ & $31(88.57 \%)$ & \\
\hline
\end{tabular}


showed that there was a significant difference between them $(P$ value $=0.03)$, with platelet counts being higher in patients with rheumatoid arthritis than in the healthy controls; however, there was no significant difference between the healthy controls, remission, and newly diagnosed RA groups.

The results showed that patients with higher disease activity had higher levels of MPV than new cases and the remission group ( $P$ value $<0.001)$, while there was no difference between the remission and newly diagnosed group in MPV (Figure 1).

Spearman correlation analysis showed a direct relationship between MPV variables and RA severity. The coeffi- cient $r$ was 0.27 and sig was $=0.005$, indicating a relatively weak and direct correlation between the two variables.

A significant difference was observed between the remission and the newly diagnosed groups $(P$ value $<0.001)$ as well as between the remission and active groups ( $P$ value $=0.001)$ in terms of erythrocyte sedimentation rate (ESR). There was also a significant difference between the remission and newly diagnosed groups $(P$ value $=0.003)$ as well as between the remission and active groups $(P$ value $=$ 0.03 ) in terms of C-reactive protein (CRP. (Table 2).

The comparison of neutrophil percentages showed that there was a significant difference between the active and the remission groups $(P$ value $=0.01)$.
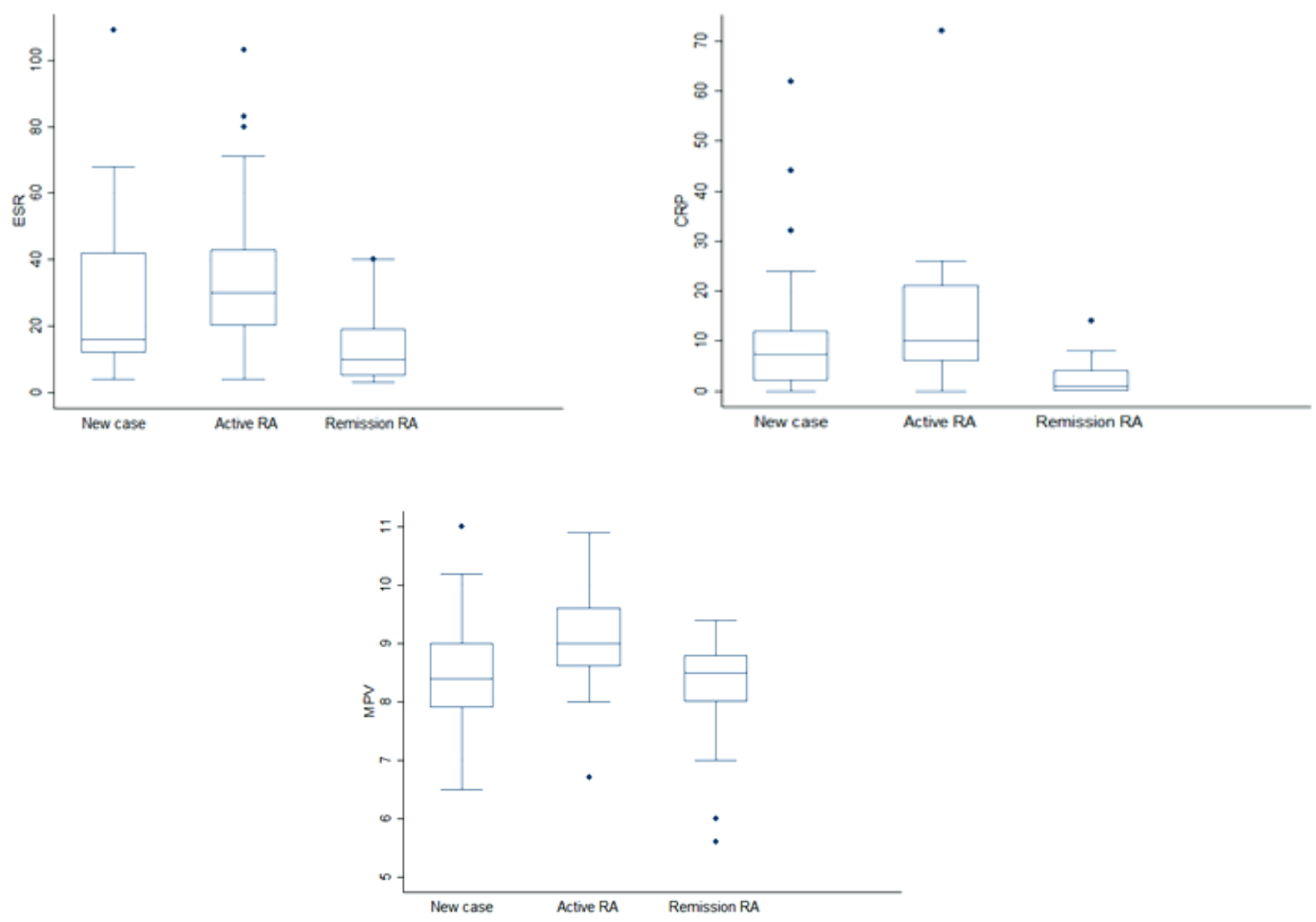

Figure 1. Distribution of erythrocyte sedimentation rate, C-reactive protein, and mean platelet volume 
Table 2. Mean of laboratory data in different groups

\begin{tabular}{|c|c|c|c|c|c|c|c|c|c|}
\hline & \multicolumn{2}{|c|}{ Healthy control } & \multicolumn{2}{|c|}{ Active rheumatoid arthritis } & \multicolumn{2}{|c|}{$\begin{array}{c}\text { Newly diagnosed rheuma- } \\
\text { toid arthritis }\end{array}$} & \multicolumn{2}{|c|}{$\begin{array}{c}\text { Remission rheumatoid } \\
\text { arthritis }\end{array}$} & \multirow[t]{2}{*}{ P-value } \\
\hline & Mean & Range & Mean & Range & Mean & Range & Mean & Range & \\
\hline $\mathrm{ESR}^{*}$ & $12 \pm 6.5$ & $8-23$ & $30 \pm 17.32$ & $20-43$ & $16 \pm 9.67$ & $12-42$ & $10 \pm 5.3$ & $5-19$ & $<0.0005$ \\
\hline CRP* & $0.62 \pm 0.09$ & $0-2.4$ & $10 \pm 4.54$ & $6-21$ & $7.4 \pm 3.54$ & $2-12$ & $1 \pm 0.6$ & $0-4$ & $<0.0005$ \\
\hline $\mathrm{WBC}^{*}$ & $5.6 \pm 2.87$ & $4.6-8.9$ & $8.3 \pm 3.2$ & $6.9-10.5$ & $7.3 \pm 1.3$ & $8-8.6$ & $6.9 \pm 2.87$ & $6.3-8.6$ & 0.058 \\
\hline $\mathrm{Hb}^{* *}$ & $13.1 \pm 1.45$ & $12.4-14$ & $12.29 \pm 1.15$ & $11.5-13.56$ & $12.93 \pm 1.44$ & $11.9-13.8$ & $12.81 \pm 1.34$ & $12.1-13.4$ & 0.107 \\
\hline PLT** & $293 \pm 42.5$ & $256-401$ & $326 \pm 56.8$ & $235-397$ & $277 \pm 34.1$ & $221-345$ & $257 \pm 24.09$ & $213-299$ & 0.037 \\
\hline $\mathrm{MPV}^{* *}$ & $8.1 \pm 1.15$ & $7.9-8.8$ & $9 \pm 1.89$ & $8.6-9.6$ & $8.6 \pm 2.09$ & $7.9-9$ & $8.5 \pm 1.34$ & $8-8.8$ & $<0.0005$ \\
\hline Neut** & $56.7 \pm 8.09$ & 54- 61 & $66.05 \pm 11.06$ & $64-72$ & $60.57 \pm 9.66$ & $57-63$ & $59.09 \pm 8.66$ & $57-61$ & $<0.0005$ \\
\hline Lymph $^{* *}$ & $36.2 \pm 8.56$ & $35-43$ & $29.88 \pm 10.06$ & $27-33.7$ & $33.4 \pm 8.72$ & $31.4-37$ & $34.14 \pm 8.25$ & $32-38.3$ & 0.11 \\
\hline
\end{tabular}

Eerythrocyte sedimentation rate (ESR); C-reactive protein (CRP); White blood cell count (WBC); Platelet number (PLT); Mean platelet volume (MPV); Hhemoglobin $(\mathrm{Hb})$;

* Kruskal-Wallis test, $\quad$ **ANOVA test

\section{Discussion}

Hematological markers associated with platelet function are often neglected. In the present study, the platelet indices were measured in patients with active RA, newly diagnosed RA patients, and RA patients in remission. Based on the results of the present study, the mean values of ESR, CRP, PLT, MPV, and neutrophils were higher in patients with active RA compared with those in patients newly diagnosed with RA, patients in remission, and the healthy controls. Moreover, the mean values of these factors were higher in the newly diagnosed RA group compared with those in the RA patients in remission. No relationship was observed between disease activity and $\mathrm{Hb}$, lymphocyte number, and WBC count.

Similarly, Yildirim et al. demonstrated that MPV was significantly higher in the RA group when compared with the healthy controls, but contrary to the current results, their results indicated a negative relationship between MPV and disease activity [17].

Yazici et al. showed that MPV and PLT values were higher in RA subjects in comparison with healthy controls. Moreover, WBC, ESR, and CRP levels were higher in RA patients than in the control group [9]. Based on the findings of the present study, high MPV levels were observed in patients with active RA compared with newly diagnosed RA patients and RA patients in remission. A lower Hb lev- el was reported by Yazici et al. in RA patients in comparison with the control group; however, in the present study, no difference was observed in $\mathrm{Hb}$ factor.

Yazici et al. demonstrated that MPV and PLT count were correlated with disease activity diagnosed by DAS28 score. Similar to the results of Yazici, the present study used DAS28 score to detect patients in remission and those with active RA. The findings of Milovanovic et al. indicated increased PLT and MPV values in patients with active RA, which decreased after 2 years of anti-TNF- $\alpha$ therapy [15]. The sample size in the present study was larger than those of the aforementioned studies. However, the aforementioned results are in line with the findings of the present study.

Contradictory to the results of the present study, Kisacik et al. indicated that MPV levels were significantly lower in patients with active RA than in the control group [16].

Nonetheless, the data obtained from the aforementioned study should not be considered as certain due to its low sample size and retrospective nature. These conflicting findings might be partially explained by different sample characteristics, such as age, sample size, and different diagnostic criteria.

The relationship between other disease activity markers such as ESR and CRP levels and disease activity in RA patients was rejected based on the findings of other studies 
$[15,18]$; however, in the present study, ESR and CRP values were higher in patients with active RA in comparison with those in patients with newly diagnosed RA and in the remission group.

The main goal of the treatment process of RA is to suppress local and systemic inflammation. Biological TNF- $\alpha$ blocking agents are the main agents for the suppression of inflammation and management of disease activity in RA patients. These medications exclusively or in combination with disease-modifying anti-rheumatic drugs can be used for the suppression of pro-inflammatory cytokines and acute-phase reactants [19]. Diverse inflammatory biomarkers are involved in the pathophysiology of RA. Based on the results of a study carried out by Caporali et al., a three-month treatment with anti-TNF- $\alpha$ leads to changes in MPV, disease activity, ESR, and CRP as well as a reduction in platelet count [20].

An inverse relationship is indicated between the changes in platelet count and platelet size due to TNF- $\alpha$ therapy [21]. A tendency to smaller sized platelets in RA patients is related to high disease activity. The small size of platelets in RA patients in the active stage was demonstrated by Kisacik et al. [16]. The findings of another study showed the opposite direction of MPV changes and its dual pathophysiological role in RA [15].

The MPV values might indicate the intensity of the inflammatory process in the active stage of RA. The overproduction of pro-inflammatory cytokines and acute-phase reactants leads to the suppression of platelet size by affecting megakaryopoiesis and the consecutive release of small-sized platelets from bone marrow. The intensive consumption of large platelets at inflammation sites may be another explanation for the reduced size of circulating platelets in active RA. In the acute stage of inflammation, large platelets have higher activity in releasing pro-inflammatory and thrombotic agents than smaller-sized platelets [21].

MPV has been well examined compared with the other variables of platelet function produced by automated blood cell counters. An association between high MPV values and increased risk of thrombotic events has been shown in several studies [22-24]. The MPV, an inexpensive test, can be used for the evaluation of disease activity in patients with RA $[25,26]$.

Limitations and Recommendations

The small sample size and short follow-up periods were the main limitations of this study. Therefore, the results of this study cannot be generalized to other populations. Larger studies with larger sample sizes should be performed to obtain more accurate results.

\section{Conclusion}

MPV and platelet numbers are higher in patients with active RA than in the healthy controls or in RA patients in remission. The findings of this study emphasize the use of MPV as a marker of high-grade inflammation for monitoring treatment in RA patients. Future prospective studies with large sample sizes are required to determine the associations between high and low values of MPV and inflammation markers.

\section{Acknowledgments}

The authors extend their gratitude to the Deputy of Research and Technology as well as all members and experts of the Research Council of Ferdowsi University for their approval of the project proposal. We would also like to thank all of the students and the Psychology School for their cooperation in the present study.

\section{Conflict of Interest}

The authors declare no conflicts of interest 


\section{References}

1. Cross M, Smith E, Hoy D, Carmona L, Wolfe F, Vos T, et al. The global burden of rheumatoid arthritis: estimates from the global burden of disease 2010 study. Ann Rheum Dis 2014;73(7):1316-22. doi: 10.1136/annrheumdis-2013-204627.

2. Alamanos Y, Drosos AA. Epidemiology of adult rheumatoid arthritis. Autoimmun Rev 2005;4(3):130-36. doi: 10.1016/j.autrev.2004.09.002.

3. Smolen JS, Aletaha D, Redlich K. The pathogenesis of rheumatoid arthritis: new insights from old clinical data? Nat Rev Rheumatol 2012;8(4):235-43. doi: 10.1038/nrrheum.2012.23.

4. Semple JW, Italiano JE, Freedman J. Platelets and the immune continuum. Nat Rev Immunol 2011;11(4):264-74. doi: $10.1038 /$ nri2956.

5. Varenhorst C, Alström U, Scirica BM, Hogue CW, Åsenblad N, Storey RF, et al. Factors contributing to the lower mortality with ticagrelor compared with clopidogrel in patients undergoing coronary artery bypass surgery. $\boldsymbol{J}$ Am Coll Cardiol 2012;60(17):1623-30. doi: 10.1016/j. jacc.2012.07.021.

6. Varenhorst C, Alström U, Braun OÖ, Storey RF, Mahaffey KW, Bertilsson M, et al. Causes of mortality with ticagrelor compared with clopidogrel in acute coronary syndromes. Heart 2014;100(22):1762-69. doi: 10.1136/ heartjnl-2014-305619.

7. Akinosoglou K, Alexopoulos D. Use of antiplatelet agents in sepsis: a glimpse into the future. Thromb Res 2014;133(2):131-38. doi: 10.1016/j.thromres.2013.07.002.

8. 8. Thomas MR, Storey RF. The role of platelets in inflammation. Thromb Haemost 2015;114(09):449-58. doi: 10.1160/TH14-12-1067.

9. Yazici S, Yazici M, Erer B, Erer B, Calik Y, Ozhan H, et al. The platelet indices in patients with rheumatoid arthritis: mean platelet volume reflects disease activity. Platelets 2010;21(2):122-25. doi: 10.3109/09537100903474373.

10. Gonzalez-Gay MA, Gonzalez-Juanatey C, Lopez-Diaz MJ, Piñeiro A, Garcia-Porrua C, Miranda-Filloy JA, et al. HLA-DRB1 and persistent chronic inflammation contribute to cardiovascular events and cardiovascular mortality in patients with rheumatoid arthritis. Arthritis Rheum 2007;57(1):125-32. doi: 10.1002/art.22482.

11. Maradit-Kremers H, Nicola PJ, Crowson CS, Ballman KV, Gabriel SE. Cardiovascular death in rheumatoid arthritis: a population-based study. Arthritis Rheum 2005;52(3):72232. doi: 10.1002/art.20878.

12. Pasceri V, Yeh ET. A tale of two diseases: atherosclerosis and rheumatoid arthritis. Circulation 1999;23(100):212426. doi: 10.1161/01.cir.100.21.2124.

13. Bathon JM, Martin RW, Fleischmann RM, Tesser JR, Schiff MH, Keystone EC, et al. A comparison of etanercept and methotrexate in patients with early rheumatoid arthritis. $\boldsymbol{N}$ Engl J Med 2000;343(22):1586-93. doi: 10.1056/NEJM200011303432201.

14. Breedveld F, Dayer J. Leflunomide: mode of action in the treatment of rheumatoid arthritis. Ann Rheum Dis 2000;59(11):841-9. doi: 10.1136/ard.59.11.841.

15. Milovanovic M, Nilsson E, Järemo P. Relationships between platelets and inflammatory markers in rheumatoid arthritis. Clin Chim Acta 2004;343(1-2):237-40. doi: 10.1016/j.ccen.2003.12.030.

16. Kisacik B, Tufan A, Kalyoncu U, Karadag O, Akdogan A, Ozturk MA, et al. Mean platelet volume (MPV) as an inflammatory marker in ankylosing spondylitis and rheumatoid arthritis. Joint Bone Spine 2008;75(3):291-4. doi: 10.1016/j.jbspin.2007.06.016.

17. Yildirim A, Karabiber M, Surucu GD, Türkbeyler IH, Karakoyun A, Selkuc MY, et al. The changes of mean platelet volume and platelet distribution width in patients with rheumatoid arthritis and their correlation with disease activity. Acta Med Medit 2015;31:1105-11.

18. Aletaha D, Nell VP, Stamm T, Uffmann M, Pflugbeil S, Machold K, et al. Acute phase reactants add little to composite disease activity indices for rheumatoid arthritis: validation of a clinical activity score. Arthritis Res Ther 2005;7(4):R796-06. doi: 10.1186/ar1740.

19. Wolfe F. Comparative usefulness of C-reactive protein and erythrocyte sedimentation rate in patients with rheumatoid arthritis. J Rheumatol 1997;24(8):1477-85.

20. Caporali R, Pallavicini FB, Filippini M, Gorla R, Marchesoni A, Favalli EG, et al. Treatment of rheumatoid arthritis with anti-TNF-alpha agents: a reappraisal. Autoimmun Rev 2009;8(3):274-80. doi: 10.1016/j.autrev.2008.11.003.

21. Gasparyan AY, Sandoo A, Stavropoulos-Kalinoglou A, Kitas GD. Mean platelet volume in patients with rheumatoid arthritis: the effect of anti-TNF-alpha therapy. Rheumatol Int 2010;30(8):1125-29. doi: 10.1007/s00296-009-1345-1.

22. Papanas N, Symeonidis G, Maltezos E, Mavridis G, Karavageli E, Vosnakidis T, et al. Mean platelet volume in patients with type 2 diabetes mellitus. Platelets 2004;15(8):475-78. doi: 10.1080/0953710042000267707.

23. Boos CJ, Beevers GD, Lip GY. Assessment of platelet activation indices using the ADVIATM 120 amongst 'highrisk'patients with hypertension. Ann Med 2007;39(1):7278. doi: 10.1080/07853890601040063.

24. Tavil Y, Sen N, Yazıcı HU, Hızal F, Abacı A, Cengel A. Mean platelet volume in patients with metabolic syndrome and its relationship with coronary artery disease. Thromb Res 2007;120(2):245-50. doi: 10.1016/j.thromres.2006.10.005.

25. Yilmaz MB, Cihan G, Guray Y, Guray U, Kisacik HL, Sasmaz H, et al. Role of mean platelet volume in triagging acute coronary syndromes. J Thromb Thrombolysis 2008;26(1):49-54. doi: 10.1007/s11239-007-0078-9.

26. Bath P, Algert C, Chapman N, Neal B. Association of mean platelet volume with risk of stroke among 3134 individuals with history of cerebrovascular disease. Stroke 2004;35(3):622-26. doi: 10.1161/01. STR.0000116105.26237.EC. 